\title{
Yang-Mills observables: from KMOC to eikonal through EFT
}

\author{
Leonardo de la Cruz, ${ }^{a, b}$ Andres Luna ${ }^{c}$ and Trevor Scheopner ${ }^{c}$ \\ a Dipartamento di Fisica e Astronomia "Augusto Righi", Università di Bologna, \\ via Irnerio 46, I-40126 Bologna, Italy \\ ${ }^{b}$ INFN - Sezione di Bologna, \\ via Irnerio 46, I-40126 Bologna, Italy \\ ${ }^{c}$ Mani L. Bhaumik Institute for Theoretical Physics, Department of Physics and Astronomy, \\ UCLA, Los Angeles, CA 90095, U.S.A. \\ E-mail: leonardo.delacruz@unibo.it, luna@physics.ucla.edu, \\ trevor@physics.ucla.edu
}

ABSTRACT: We obtain a conservative Hamiltonian describing the interactions of two charged bodies in Yang-Mills through $\mathcal{O}\left(\alpha^{2}\right)$ and to all orders in velocity. Our calculation extends a recently-introduced framework based on scattering amplitudes and effective field theory (EFT) to consider color-charged objects. These results are checked against the direct integration of the observables in the Kosower-Maybee-O'Connell (KMOC) formalism. At the order we consider we find that the linear and color impulses in a scattering event can be concisely described in terms of the eikonal phase, thus extending the domain of applicability of a formula originally proposed in the context of spinning particles.

KEywords: Scattering Amplitudes, Effective Field Theories, Perturbative QCD

ArXiv EPrint: 2108.02178 


\section{Contents}

1 Introduction 1

2 KMOC approach to color observables $\quad 3$

2.1 Leading order 4

2.2 Next-to-Leading-Order 5

3 Hamiltonian approach to color dynamics $\quad 8$

$\begin{array}{lll}3.1 \text { Classical perturbation theory } & 8\end{array}$

$\begin{array}{ll}3.2 & \text { Hamiltonian from effective field theory }\end{array}$

$\begin{array}{lll}3.2 .1 & \text { Full theory amplitudes from unitarity } & 10\end{array}$

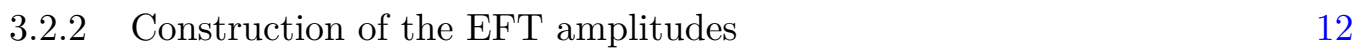

$\begin{array}{lll}3.3 & \text { Observables from the eikonal phase } & 14\end{array}$

4 Conclusions $\quad 16$

\section{Introduction}

The Kosower-Maybee-O'Connell (KMOC) formalism [1-3] is a first principle approach to extract the classical limit, understood as the limit $\hbar \rightarrow 0$, from on-shell scattering amplitudes. It is based on the construction of certain observables which are well-defined at the quantum and classical levels. They can be defined by considering the expectation value of certain operators $\mathbb{O}$ evaluated at the beginning and at the end of the scattering event. Considering the two-to-two classical scattering the observable associated with the operator $\mathbb{O}$ is given by

$$
\langle\Delta O\rangle=\left\langle\Psi\left|S^{\dagger} \mathbb{O} S\right| \Psi\right\rangle-\langle\Psi|\mathbb{O}| \Psi\rangle
$$

where $S=1+i T$. The "in" states $|\Psi\rangle$ are two-particle coherent states for momentum and color, whose function is to give the notion of point particles with a sharply-defined position, momenta, and color. To make this notion precise, the restoration of $\hbar$ 's on couplings and color factors as well as the distinction between momenta $p$ and wavenumber $\bar{p}$ for certain particles play an important role.

Employing unitarity, the observables can be written as

$$
\langle\Delta O\rangle=i\langle\Psi|[\mathbb{O}, T]| \Psi\rangle+\left\langle\Psi\left|T^{\dagger}[\mathbb{O}, T]\right| \Psi\right\rangle
$$

which can be used to derive general expressions for these observables in terms of amplitudes. In this paper we will consider the color charge operator $\mathbb{C}_{1}^{a}$ and the momentum operator $\mathbb{P}_{1}^{\mu}$ of one of the particles, but of course the other particle can be chosen as well. The observables associated to these operators are called the color impulse $\Delta c_{1}^{a}$ and the momentum 
impulse $\Delta p_{1}^{\mu}$. The KMOC formalism has been applied to the study of waveforms [4], soft theorems [5], radiative gravitational observables at two-loops [6, 7] and adapted to study the classical limit of thermal currents [8].

On the other hand, the classical limit can also be described in the language of effective field theory (EFT). This idea was pioneered in ref. [9], which proposed the application of the well-established scattering-amplitudes toolkit to the derivation of gravitational potentials. Later, an EFT of non-relativistic scalar fields was developed [10], and used to translate a one-loop scattering amplitude into the $\mathcal{O}\left(G^{2}\right)$ canonical Hamiltonian, which is equivalent to the results of Westpfahl [11]. This approach was later implemented to obtain novel results at $\mathcal{O}\left(G^{3}\right)$ order $[12-14]$.

Besides making use of the KMOC formalism or non-relativistic EFTs, various approaches have been developed to extract the dynamics of compact objects from scattering data. These include making use of the Lippman-Schwinger equation [15, 16], a heavy black hole effective theory and its generalizations [17-20], developing a boundary-to-bound (B2B) dictionary [21, 22], implementing a post-Minkowskian EFT [23-25] and a worldline QFT [26]. More recently the conservative binary potential at $\mathcal{O}\left(G^{4}\right)$ was obtained by means of an amplitude-action relation that allows the calculation of physical observables directly from the scattering amplitude [27].

The techniques mentioned above have been extended in multiple directions in recent years, including the computation of observables in supergravity [28-30] and other generalizations of general relativity [31, 32], the study of three-body dynamics [33], incorporating the radiation emitted by the binary into their analysis [34-39], and considering tidal deformations [40-46] and spin effects [47-63] of the astrophysical objects.

A further relation between amplitudes and classical observables is given through the eikonal phase, which is obtained as the Fourier transform to impact parameter space of the scattering amplitude [64]. In turn, one can derive the scattering angle through differentiation of the eikonal phase. This subject has seen renewed interest [29, 30, 65-74] and a recent calculation in ref. [56] showed a surprising structure for the expression of the observables in terms of the eikonal phase. This formula was the first example of such a relation for arbitrary orientations of the spins. ${ }^{1}$ This striking observation potentially implies that all physical observables are obtainable via simple manipulations of the scattering amplitude.

While most of the attention has been given to gravitational theories, Yang-Mills theory shares many important physical features with gravity, like non-linearity and a gauge structure. Furthermore, the double copy relates scattering amplitudes in both theories. ${ }^{2}$ The connection has showed to be deeper than this, holding in a classical worldline setting [7888], and extending to exact maps [89, 90]. ${ }^{3}$ Then, since perturbation theory in Yang-Mills is far simpler than in standard approaches of gravity, one may study Yang-Mills as a toy model for gravitational dynamics or as a building block that could be double copied to gravity. One may also note that, as already pointed out in ref. [3], the dynamics of the

\footnotetext{
${ }^{1}$ Before this, there was evidence for such a relation in the special kinematic configuration where the spins of the particles are parallel to the angular momentum of the system [50,75, 76].

${ }^{2}$ The double copy has been reviewed thoroughly in ref. [77].

${ }^{3}$ The classical double copy has also made contact with fluid dynamics, as shown in refs. [91, 92].
} 
color degrees of freedom in Yang-Mills, is in many respects analogous to spin (though actually simpler). This analogy with spin will be evidenced in a generalization of the formula of ref. [56], now describing the dynamics of color charges.

The proliferation of approaches to extract classical information from quantum scattering amplitudes motivates us to strive for an understanding of the relations between them. The goal of this paper is to use Yang-Mills theory as a toy model to study the connection between three such approaches. Namely, the KMOC formalism, the Hamiltonian approach to classical dynamics, and a formula directly relating the eikonal phase with classical observables.

The remainder of this paper is structured as follows: in section 2 we compute color and momentum impulse at NLO (Next-to-Leading Order) using the integrands obtained in ref. [3]. Then, In section 3, we develop the Hamiltonian approach to classical dynamics. First, we show the necessary full-theory amplitudes and use a matching procedure to an EFT to obtain the desired two-body Hamiltonian. Then we use the derived Hamiltonian to compute scattering observables, and check their match both to the KMOC approach of section 2, as well as to the conjecture of ref. [56], which directly relates these observables to the eikonal phase, and holds (almost unalteredly) when we include color effects. We present our concluding remarks in section 4 .

\section{KMOC approach to color observables}

In this section we introduce the KMOC approach for color and introduce our notation and conventions. The classical scattering of two color-charged scalar particles of masses $m_{1}$ and $m_{2}$ can be modeled by the action

$$
S=\int \hat{\mathrm{d}}^{4} x\left[\sum_{i=1,2}\left(\left(D_{\mu} \varphi_{i}\right)^{\dagger}\left(D^{\mu} \varphi_{i}\right)-\frac{m_{i}^{2}}{\hbar^{2}} \varphi_{i}^{\dagger} \varphi_{i}\right)-\frac{1}{4} F_{\mu \nu}^{a} F^{a \mu \nu}\right],
$$

where $D_{\mu}=\partial_{\mu}+i g A_{\mu}^{a} T_{R}^{a}$ and $\hat{\mathrm{d}}^{n} x=\left(\mathrm{d}^{n} x\right) /(2 \pi)^{n}$. The generators $T_{a}^{R}$ of the Lie algebra of $\mathrm{SU}(N)$ are in some representation $R$. The color charge operators, obtained from the Noether procedure, satisfy the usual Lie algebra modified by a factor of $\hbar$

$$
\left[\mathbb{C}^{a}, \mathbb{C}^{b}\right]=i \hbar f^{a b c} \mathbb{C}^{c},
$$

emphasizing that $\mathbb{C}^{a}$ corresponds to an operator and

$$
\left\langle p_{i}\left|\mathbb{C}^{a}\right| p^{j}\right\rangle \equiv\left(C^{a}\right)_{i}^{j}=\hbar\left(T_{R}^{a}\right)_{i}^{j} .
$$

So the color factors $\left(C^{a}\right)_{i}^{j}$ are simply rescalings of the usual generators $\left(T_{R}^{a}\right)_{i}^{j}$. The classical color charges are then defined by

$$
c^{a} \equiv\left\langle\psi\left|\mathbb{C}^{a}\right| \psi\right\rangle,
$$

where the states $|\psi\rangle$ are coherent states for $\mathrm{SU}(N)$, whose explicit form will not be relevant for our purposes. ${ }^{4}$ These states ensure the correct behavior of color charges in the classical

\footnotetext{
${ }^{4}$ When considering the classical limit of multi-particle states, the full state is a tensor product of coherent states for the kinematics and coherent states for color. $\mathrm{SU}(N)$ coherent states can be constructed using Schwinger bosons. For the kinematic part, these states can be understood as coherent states for a "firstquantized" particle for the restricted Poincaré group. See ref. [3] for details.
} 
limit, namely

$$
\begin{aligned}
\left\langle\psi\left|\mathbb{C}^{a}\right| \psi\right\rangle & =\text { finite }, \\
\left\langle\psi\left|\mathbb{C}^{a} \mathbb{C}^{b}\right| \psi\right\rangle & =c^{a} c^{b}+\text { negligible },
\end{aligned}
$$

which is guaranteed by choosing the dimension of the representation $R$ to be large. The factors of $\hbar$ in eq. (2.2) produce a nontrivial interplay between color factors and kinematics in the classical limit. However ultimately classical quantities do not have any factors of $\hbar$ as it should be. Thus, for the purposes of this paper we will quote the integrands derived in ref. [3] dropping the bar notation for wavenumbers. We will also employ the notation $\Delta O^{(L)}$ to indicate the $L$-loop contribution to the observable such that the full result is given by

$$
\Delta O=\Delta O^{(0)}+\Delta O^{(1)}+\ldots .
$$

We also introduce the following notation for the Dirac-delta

$$
\hat{\delta}(x)=2 \pi \delta(x), \quad \hat{\delta}^{\prime}(x)=\frac{i}{(x-i \epsilon)^{2}}-\frac{i}{(x+i \epsilon)^{2}} .
$$

\subsection{Leading order}

Let us briefly review the LO calculation of ref. [3] in order to introduce some notation. We define the integral

$$
\mathcal{I}_{\succ} \equiv \int \hat{\mathrm{d}}^{4} q \frac{\hat{\delta}\left(q \cdot u_{1}\right) \hat{\delta}\left(q \cdot u_{2}\right)}{q^{2}} e^{-i q \cdot b}
$$

where $p_{i}^{\mu}=m_{i} u_{i}^{\mu}$ and $b^{\mu}$ is the impact parameter. Recalling that $b^{\mu}$ is spacelike we also define $|b| \equiv \sqrt{-b^{2}}$. The classical four velocities $u_{i}$ are normalized to $u_{i}^{2}=1$. The divergent integral $I_{\succ}$ can be regulated using a cut-off regulator ${ }^{5} L$

$$
\mathcal{I}_{\succ \prec}=\frac{1}{4 \pi \sqrt{\sigma^{2}-1}} \log \left(\frac{|b|^{2}}{L^{2}}\right),
$$

where $\sigma$ is the standard Lorentz factor $\sigma=u_{1} \cdot u_{2}$. The LO momentum impulse can then be written as

$$
\Delta p_{1}^{(0), \mu}=-g^{2} \sigma c_{1} \cdot c_{2} \frac{\partial \mathcal{I}_{\succ}}{\partial b_{\mu}}
$$

where $c_{1} \cdot c_{2} \equiv c_{1}^{a} c_{2}^{a}$. So the momentum impulse is given by

$$
\Delta p_{1}^{(0), \mu}=-2 \alpha c_{1} \cdot c_{2} \frac{\sigma}{\sqrt{\sigma^{2}-1}} \frac{b^{\mu}}{b^{2}},
$$

where $\alpha \equiv g^{2} /(4 \pi)$. Similarly the color impulse at leading order reads

$$
\Delta c_{1}^{(0), a}=g^{2} \sigma f^{a b c} c_{1}^{b} c_{2}^{c} \mathcal{I}_{\succ}=\alpha f^{a b c} c_{1}^{b} c_{2}^{c} \frac{\sigma}{\sqrt{\sigma^{2}-1}} \log \left(\frac{|b|^{2}}{L^{2}}\right) .
$$

The divergence of the color impulse is the familiar divergence due to the long-range nature of $1 / r^{2}$ forces in four-dimensions.

\footnotetext{
${ }^{5}$ To obtain this result we have set up a differential equation for $I_{\succ}$ instead of explicitly including a regulator $L$ on the integration region. $I_{\succ}$ satisfies $b_{\mu} \partial I_{\succ} / \partial b_{\mu}=1 /\left(2 \pi \sqrt{\sigma^{2}-1}\right)$.
} 


\subsection{Next-to-Leading-Order}

The NLO momentum impulse can be obtained from the QED one computed in ref. [1] using the charge to color replacements $Q_{1} Q_{2} \rightarrow c_{1} \cdot c_{2}$ and $e \rightarrow g$. That this replacement works follows from the color-decomposition of the QCD amplitude and $\hbar$-counting as detailed in [3]. The result reads

$$
\begin{aligned}
\Delta p_{1}^{\mu,(1)}= & i \frac{g^{4}\left(c_{1} \cdot c_{2}\right)^{2}}{2} \int \hat{\mathrm{d}}^{4} \ell \hat{\mathrm{d}}^{4} q \frac{\hat{\delta}\left(u_{1} \cdot q\right) \hat{\delta}\left(u_{2} \cdot q\right)}{\ell^{2}(\ell-q)^{2}} e^{-i q \cdot b}\left[q ^ { \mu } \left\{\frac{\hat{\delta}\left(u_{2} \cdot \ell\right)}{m_{1}}+\frac{\hat{\delta}\left(u_{1} \cdot \ell\right)}{m_{2}}\right.\right. \\
& \left.+\left(u_{1} \cdot u_{2}\right)^{2} \ell \cdot(\ell-q)\left(\frac{\hat{\delta}\left(u_{1} \cdot \ell\right)}{m_{2}\left(u_{2} \cdot \ell-i \epsilon\right)^{2}}+\frac{\hat{\delta}\left(u_{2} \cdot \ell\right)}{m_{1}\left(u_{1} \cdot \ell+i \epsilon\right)^{2}}\right)\right\} \\
& \left.-i\left(u_{1} \cdot u_{2}\right)^{2} \ell^{\mu} \ell \cdot(\ell-q)\left(\frac{\hat{\delta}^{\prime}\left(u_{1} \cdot \ell\right) \hat{\delta}\left(u_{2} \cdot \ell\right)}{m_{1}}-\frac{\hat{\delta}\left(u_{1} \cdot \ell\right) \hat{\delta}^{\prime}\left(u_{2} \cdot \ell\right)}{m_{2}}\right)\right] .
\end{aligned}
$$

On the other hand the NLO color impulse is given by

$$
\begin{aligned}
\Delta c_{1}^{a,(1)}= & g^{4} \int \hat{\mathrm{d}}^{4} q \hat{\mathrm{d}}^{4} \ell \hat{\delta}\left(u_{1} \cdot q\right) \hat{\delta}\left(u_{2} \cdot q\right) e^{-i q \cdot b} \frac{1}{\ell^{2}(\ell-q)^{2}} \\
& \times\left\{\hat { \delta } ( u _ { 1 } \cdot \ell ) \left[\frac { f ^ { a c d } c _ { 1 } ^ { c } c _ { 2 } ^ { d } ( c _ { 1 } \cdot c _ { 2 } ) } { m _ { 2 } } \left[1+\left(u_{1} \cdot u_{2}\right)^{2} \ell \cdot(\ell-q)\left(\frac{1}{\left(u_{2} \cdot \ell-i \epsilon\right)^{2}}\right.\right.\right.\right. \\
& \left.\left.\left.+i \hat{\delta}^{\prime}\left(u_{2} \cdot \ell\right)\right)\right]-f^{a c d} f^{d b e} c_{1}^{b} c_{1}^{c} c_{2}^{e} \frac{\left(u_{1} \cdot u_{2}\right)^{2}}{2} \hat{\delta}\left(u_{2} \cdot \ell\right)\right] \\
& +\hat{\delta}\left(u_{2} \cdot \ell\right)\left[\frac { f ^ { a c d } c _ { 1 } ^ { c } c _ { 2 } ^ { d } ( c _ { 1 } \cdot c _ { 2 } ) } { m _ { 1 } } \left[1+\left(u_{1} \cdot u_{2}\right)^{2} \ell \cdot(\ell-q)\left(\frac{1}{\left(u_{1} \cdot \ell+i \epsilon\right)^{2}}\right.\right.\right. \\
& \left.\left.\left.\left.-i \hat{\delta}^{\prime}\left(u_{1} \cdot \ell\right)\right)\right]+f^{a c d} f^{d b e} c_{1}^{e} c_{2}^{b} c_{2}^{c} \frac{\left(u_{1} \cdot u_{2}\right)^{2}}{2} \hat{\delta}\left(u_{1} \cdot \ell\right)\right]\right\}
\end{aligned}
$$

Inspecting eqs. (2.14) and (2.15) it is easy to see that the color and momentum impulses can be expressed in terms of the following "master integrals"

$$
\begin{aligned}
\mathcal{I}_{\triangle}^{i}[\alpha, \beta, \gamma] & =\int \hat{\mathrm{d}}^{4} q \hat{\delta}\left(u_{1} \cdot q\right) \hat{\delta}\left(u_{2} \cdot q\right) e^{-i q \cdot b} \int \hat{\mathrm{d}}^{4} \ell \frac{\hat{\delta}\left(u_{i} \cdot \ell\right)}{\left[\ell^{2}\right]^{\alpha}\left[(\ell-q)^{2}\right]^{\beta}\left[\left(\ell \cdot u_{j}+(-1)^{i} i \epsilon\right)\right]^{\gamma}}, \quad j \neq i \\
\mathcal{I}_{\text {III }}[\alpha, \beta] & =\int \hat{\mathrm{d}}^{4} q \hat{\delta}\left(u_{1} \cdot q\right) \hat{\delta}\left(u_{2} \cdot q\right) e^{-i q \cdot b} \int \hat{\mathrm{d}}^{4} \ell \frac{\hat{\delta}\left(u_{1} \cdot \ell\right) \hat{\delta}\left(u_{2} \cdot \ell\right)}{\left[\ell^{2}\right]^{\alpha}\left[(\ell-q)^{2}\right]^{\beta}},
\end{aligned}
$$

where the vector dependence on the momentum transfer $q^{\mu}$ can be recovered by taking derivatives w.r.t. the impact parameter $b^{\mu}$. Notice that we have excluded from the master integrals those involving $\hat{\delta}^{\prime}(x)$ since they can be reduced to the above cases using the identity

$$
\int \hat{\mathrm{d}} x x \hat{\delta}^{\prime}(x) f\left(x^{2}\right)=-\int \hat{\mathrm{d}} x \hat{\delta}(x) f\left(x^{2}\right)
$$

Following arguments by Kälin-Porto [23], the integrals below vanish due to the presence of a double pole on a convergent integral ${ }^{6}$

$$
\mathcal{I}_{\triangle}^{i}[1,1,2]=\mathcal{I}_{\triangle}^{i}[0,1,2]=\mathcal{I}_{\triangle}^{i}[1,0,2]=0, \quad i=1,2,
$$

\footnotetext{
${ }^{6}$ This result can also be shown by first using the Dirac-delta constraint and then IBP identities. As emphasized by Kälin-Porto these integrals do contribute in $d>4$ [69].
} 
and therefore only $I_{\triangle}^{i}[1,1,0]$ contributes to the observables. In the following we then simply write $\mathcal{I}_{\triangle}^{i}[1,1,0] \equiv \mathcal{I}_{\triangle}^{i}$ and for later purpose we write $\mathcal{I}_{\mathbb{I I}}[1,1] \equiv \mathcal{I}_{\mathbb{I}}$. We also have that

$$
\mathcal{I}_{\text {Ii[ }}[1,0]=\mathcal{I}_{\text {IiI }}[0,1]=0
$$

since their loop integrals reduce to massless tadpole integrals. Now let us move on with the reductions of integrals of the form

$$
I^{\mu}=\int \hat{\mathrm{d}}^{4} \ell \ell^{\mu} \ell \cdot(\ell-q) \frac{\hat{\delta}^{\prime}\left(u_{1} \cdot \ell\right) \hat{\delta}\left(u_{2} \cdot \ell\right)}{\ell^{2}(\ell-q)^{2}},
$$

which appear in eq. (2.14) and its mirror obtained by $1 \leftrightarrow 2$. In contrast to the above vanishing integrals, the presence of the numerator makes this integral nonzero. Let us also recall that they are still integrated over the momentum transfer $q$ and therefore in the integral reduction we can set to zero any term proportional to eq. (2.19) or (2.20). We find convenient to perform a simple Passarino-Veltman reduction on eq. (2.21) so we can write

$$
I^{\mu}=K_{1} u_{1}^{\mu}+K_{2} u_{2}^{\mu}+K_{3} q^{\mu},
$$

where setting up a system of equations the resulting coefficients are

$$
K_{1}=\frac{1}{1-\sigma^{2}} u_{1} \cdot I, \quad K_{2}=-\frac{\sigma}{1-\sigma^{2}} u_{1} \cdot I, \quad K_{3}=\frac{1}{q^{2}} q \cdot I,
$$

where we have used the delta constraints $\hat{\delta}\left(q \cdot u_{1}\right)$ and $\hat{\delta}\left(q \cdot u_{2}\right)$ on which the integral is supported. The result thus depends only on two integrals, namely $u_{1} \cdot I$ and $q \cdot I$. After cancellations, the product $q \cdot I$ leads to

$$
q \cdot I=\frac{1}{4} \int \hat{\mathrm{d}}^{4} \ell\left[\frac{2 q^{2}}{\ell^{2}}-\frac{\left(q^{2}\right)^{2}}{\ell^{2}(\ell-q)^{2}}\right] \hat{\delta}^{\prime}\left(u_{1} \cdot \ell\right) \hat{\delta}\left(u_{2} \cdot \ell\right)
$$

which can be set to zero using the defintion of $\hat{\delta}^{\prime}(x)$ and after integration over $q$ using eq. (2.19). Therefore we can express eq. (2.21) only in terms of the integral

$$
\begin{aligned}
u_{1} \cdot I & =\int \hat{\mathrm{d}}^{4} \ell u_{1} \cdot \ell \ell \cdot(\ell-q) \frac{\hat{\delta}^{\prime}\left(u_{1} \cdot \ell\right) \hat{\delta}\left(u_{2} \cdot \ell\right)}{\ell^{2}(\ell-q)^{2}} \\
& =-\int \hat{\mathrm{d}}^{4} \ell \ell \cdot(\ell-q) \frac{\hat{\delta}\left(u_{1} \cdot \ell\right) \hat{\delta}\left(u_{2} \cdot \ell\right)}{\ell^{2}(\ell-q)^{2}} .
\end{aligned}
$$

Without loss of generality, the second equality can be checked by choosing a frame where $u_{1}=(1,0,0,0)$ and $u_{2}=(\sigma, 0,0, \sigma \beta)$ and $\beta$ is defined from the condition $\sigma^{2}-\sigma^{2} \beta^{2}=1$. We can further reduce this integral ignoring vanishing terms (i.e., terms which have the form (2.20)) thus obtaining

$$
u_{1} \cdot I=\frac{1}{2} q^{2} \int \hat{\mathrm{d}}^{4} \ell \frac{\hat{\delta}\left(u_{1} \cdot \ell\right) \hat{\delta}\left(u_{2} \cdot \ell\right)}{\ell^{2}(\ell-q)^{2}} .
$$

The result for $I^{\mu}$ then reads

$$
I^{\mu}=\frac{1}{2} q^{2}\left(\frac{1}{1-\sigma^{2}} u_{1}^{\mu}-\frac{\sigma}{1-\sigma^{2}} u_{2}^{\mu}\right) \int \hat{\mathrm{d}}^{4} \ell \frac{\hat{\delta}\left(u_{1} \cdot \ell\right) \hat{\delta}\left(u_{2} \cdot \ell\right)}{\ell^{2}(\ell-q)^{2}},
$$


which implies that we can express our results only in terms of the integrals (2.16)-(2.17) as claimed. Therefore, excluding all vanishing contributions, the impulses in terms of the master integrals can be written as

$$
\begin{aligned}
\Delta p_{1}^{\mu,(1)}= & \frac{g^{4}\left(c_{1} \cdot c_{2}\right)^{2}}{2}\left\{-\frac{\partial}{\partial b_{\mu}}\left[\frac{\mathcal{I}_{\triangle}^{1}}{m_{2}}+\frac{\mathcal{I}_{\triangle}^{2}}{m_{1}}\right]\right. \\
& \left.-\left[\frac{\sigma^{2}}{2\left(1-\sigma^{2}\right)}\left(\frac{u_{1}^{\mu}}{m_{1}}-\frac{\sigma u_{2}^{\mu}}{m_{1}}\right)-(1 \leftrightarrow 2)\right] \frac{\partial}{\partial b_{\nu}} \frac{\partial}{\partial b^{\nu}} \mathcal{I}_{\text {III }}\right\}
\end{aligned}
$$

and

$$
\Delta c_{1}^{a,(1)}=g^{4}\left\{f^{a c d} c_{1}^{c} c_{2}^{d}\left(c_{1} \cdot c_{2}\right)\left(\frac{\mathcal{I}_{\triangle}^{1}}{m_{2}}+\frac{\mathcal{I}_{\triangle}^{2}}{m_{1}}\right)+\frac{\sigma^{2}}{2}\left(f^{a c d} f^{d b e} c_{1}^{e} c_{2}^{b} c_{2}^{c}-f^{a c d} f^{d b e} c_{1}^{b} c_{1}^{c} c_{2}^{e}\right) \mathcal{I}_{\text {III }}\right\} .
$$

Let us now consider the integration of the master integrals. The triangle one is wellknown (see e.g., ref. [93]) and we simply quote the result

$$
\mathcal{I}_{\triangle}^{1}=\int \hat{\mathrm{d}}^{4} q \int \hat{\mathrm{d}}^{4} \ell \hat{\delta}\left(u_{1} \cdot q\right) \hat{\delta}\left(u_{2} \cdot q\right) e^{-i q \cdot b} \frac{\hat{\delta}\left(u_{1} \cdot \ell\right)}{\ell^{2}(\ell-q)^{2}}=\frac{1}{16 \pi} \frac{1}{\sigma \beta|b|} .
$$

The loop integral inside $\mathcal{I}_{\text {III }}[1,1]$ can be computed using dimensional regularization [94], leading to

$$
\int \hat{\mathrm{d}}^{D} \ell \frac{\hat{\delta}\left(u_{1} \cdot \ell\right) \hat{\delta}\left(u_{2} \cdot \ell\right)}{\ell^{2}(\ell-q)^{2}}=\frac{1}{2 \pi \sigma \beta q^{2}}\left[\frac{1}{\varepsilon}-\log \left(-q^{2}\right)\right]
$$

where $D=4-2 \varepsilon$. The usual factor $\mu^{2 \varepsilon}$ depending on an arbitrary scale $\mu$ and $e^{\varepsilon \gamma_{E}}$ have been used to ensure the correct (integer) mass dimension of the integral and to avoid the proliferation of the Euler-Mascheroni constant $\gamma_{E}$, respectively. The divergent term leads to a contact term that can be discarded in the classical limit. ${ }^{7}$ Therefore, keeping only the finite part we have

$$
\frac{\partial}{\partial b_{\nu}} \frac{\partial}{\partial b^{\nu}} \mathcal{I}_{\text {III }}=\frac{1}{2 \pi \sigma \beta} \int \hat{\mathrm{d}}^{4} q \hat{\delta}\left(u_{1} \cdot q\right) \hat{\delta}\left(u_{2} \cdot q\right) e^{-i q \cdot b} \log \left(-q^{2}\right)=\frac{1}{2 \pi^{2} \sigma^{2} \beta^{2}} \frac{1}{b^{2}} .
$$

It will also be convenient to use a cut-off regularization to evaluate the divergent integral $\mathcal{I}_{\text {III. }}$. Exchanging the integration orders and introducing the change of variables $Q=-\ell+q$ we have

$$
\mathcal{I}_{\text {III }}=\int \hat{\mathrm{d}}^{4} \ell \frac{\hat{\delta}\left(u_{1} \cdot \ell\right) \hat{\delta}\left(u_{2} \cdot \ell\right)}{\ell^{2}} e^{-i \ell \cdot b} \int \hat{\mathrm{d}}^{4} Q \hat{\delta}\left(u_{1} \cdot Q\right) \hat{\delta}\left(u_{2} \cdot Q\right) e^{-i Q \cdot b} \frac{1}{Q^{2}},
$$

which leads to the product of two integrals of the form (2.9). Hence the result is simply

$$
\mathcal{I}_{\text {III }}=\mathcal{I}_{\succ}^{2}=\frac{1}{16 \pi^{2} \sigma^{2} \beta^{2}} \log ^{2}\left(\frac{|b|^{2}}{L^{2}}\right) .
$$

\footnotetext{
${ }^{7}$ Notice that the factor of $q^{2}$ in the denominator cancels after taking derivatives with respect to the impact parameter, so the singular term leads to $\delta^{2}(\mathbf{b})$ which we can set to zero because we assume $\mathbf{b} \neq 0$.
} 
For later purposes we will express the color impulse in terms of the cut-off regulated integral. Our full integrated result for the NLO momentum impulse then reads

$$
\begin{aligned}
\Delta p_{1}^{\mu,(1)}= & \left(c_{1} \cdot c_{2}\right)^{2} \frac{2 \pi \alpha^{2}}{m_{1} m_{2}}\left\{-\frac{1}{4 \sqrt{\sigma^{2}-1}}\left(m_{1}+m_{2}\right) \frac{b^{\mu}}{|b|^{3}}\right. \\
& \left.-\frac{1}{\pi} \frac{1}{b^{2}} \frac{\sigma^{2}}{\left(\sigma^{2}-1\right)^{2}}\left[\left(m_{2}+\sigma m_{1}\right) u_{1}^{\mu}-\left(m_{1}+\sigma m_{2}\right) u_{2}^{\mu}\right]\right\},
\end{aligned}
$$

and for the NLO color impulse

$$
\begin{aligned}
\Delta c_{1}^{a,(1)}= & \alpha^{2}\left\{\pi \frac{f^{a c d} c_{1}^{c} c_{2}^{d}\left(c_{1} \cdot c_{2}\right)}{\sqrt{\sigma^{2}-1}|b|}\left(\frac{1}{m_{1}}+\frac{1}{m_{2}}\right)\right. \\
& \left.+\frac{1}{2} \frac{\sigma^{2}}{\left(\sigma^{2}-1\right)} \log ^{2}\left(\frac{|b|^{2}}{L^{2}}\right)\left[f^{a c d} f^{d b e} c_{1}^{e} c_{2}^{b} c_{2}^{c}-f^{a c d} f^{d b e} c_{1}^{b} c_{1}^{c} c_{2}^{e}\right]\right\} .
\end{aligned}
$$

\section{Hamiltonian approach to color dynamics}

In this section we will compute the position-space Hamiltonian $H$ that describes the classical dynamics of the two-to-two scattering of SU $(N)$-colored objects with masses $m_{1}$ and $m_{2}$ and color charges $c_{1}$ and $c_{2}$. The classical dynamics described by such a Hamiltonian must be consistent with Wong's equations [95] and its perturbative solutions and by extension to observables in the KMOC formalism. Let $\boldsymbol{r}$ and $\boldsymbol{p}$ be the relative distance between the particles and the momentum vector in the center of mass frame, respectively. We are interested in a perturbative expansion of the Hamiltonian

$$
H \equiv H\left(\boldsymbol{r}, \boldsymbol{p}, \mathcal{C}_{i}\right)=\sqrt{\boldsymbol{p}^{2}+m_{1}^{2}}+\sqrt{\boldsymbol{p}^{2}+m_{2}^{2}}+V\left(\boldsymbol{r}^{2}, \boldsymbol{p}^{2}, \mathcal{C}_{i}\right)+\ldots,
$$

where the potential is an expansion up to the second power in the coupling constant $\alpha$ and the color structures $\mathcal{C}_{i}$ are all possible functions of the color charges that can appear in the amplitude. These charges are understood in the sense of Wong, i.e., as the classical limit of a quantum operator in a large representation of the gauge group so they can be treated as $c$-numbers.

\subsection{Classical perturbation theory}

Consider the general problem of an arbitrary Hamiltonian $H$ describing the interaction of two particles with color charges $c_{1}$ and $c_{2}$ in their center of mass frame. While, as usual, $\boldsymbol{r}$ and $\boldsymbol{p}$ are canonically-conjugate to each other, color charges do not have a natural canonical conjugate. To derive the equations of motion we use the fact that they satisfy the relation $[96,97]$

$$
\left\{c_{i}^{a}, c_{j}^{b}\right\}=\delta_{i j} f^{a b c} c_{i}^{c}, \quad i, j=1,2,
$$

where $\{A, B\}$ is the Poisson bracket of $A$ and $B$. The equations of motion are then

$$
\dot{\boldsymbol{r}}=\frac{\partial H}{\partial \boldsymbol{p}}, \quad \dot{\boldsymbol{p}}=-\frac{\partial H}{\partial \boldsymbol{r}}, \quad \dot{c}_{i}^{a}=f^{a b c} c_{i}^{b} \frac{\partial H}{\partial c_{i}^{c}}, \quad i=1,2 .
$$


In the color equation of motion, no summation over $i$ is implied on the right-hand side. For the purpose of finding the impulse $\Delta \boldsymbol{p}$ we find it convenient to use Cartesian coordinates. One can solve the equations of motion for coordinates, momenta, and colors as a function of time.

There are conservation laws that aid the construction of classical solutions. These fix the energy and the total angular momentum in terms of their asymptotic values. For example for the energy we have

$$
E \equiv H\left(\boldsymbol{r}_{\infty}, \boldsymbol{p}_{\infty}, c_{1}, c_{2}\right)=\sqrt{\boldsymbol{p}_{\infty}^{2}+m_{1}^{2}}+\sqrt{\boldsymbol{p}_{\infty}^{2}+m_{2}^{2}},
$$

where $\boldsymbol{p}_{\infty}=p_{\infty} \boldsymbol{e}_{z}$ is the incoming momentum at infinity. We take the orbital angular momentum at infinity to be

$$
\boldsymbol{L} \equiv \boldsymbol{b} \times \boldsymbol{p}_{\infty}=b p_{\infty} \boldsymbol{e}_{y}
$$

where $\boldsymbol{b}=-b \boldsymbol{e}_{x}$ and $b$ is the impact parameter. We solve the equations of motion perturbatively in the coupling constant, i.e. we search for a solution for coordinates, momenta, and colors of the form

$$
\begin{aligned}
\boldsymbol{r}(t) & =\boldsymbol{r}_{0}(t)+\alpha \boldsymbol{r}_{1}(t)+\alpha^{2} \boldsymbol{r}_{2}(t)+\ldots, \\
\boldsymbol{p}(t) & =\boldsymbol{p}_{0}(t)+\alpha \boldsymbol{p}_{1}(t)+\alpha^{2} \boldsymbol{p}_{2}(t)+\ldots, \\
c_{i}^{a}(t) & =c_{i, 0}^{a}(t)+\alpha c_{i, 1}^{a}(t)+\alpha^{2} c_{i, 2}^{a}(t)+\ldots .
\end{aligned}
$$

Replacing them in the equations of motion (3.3) leads to iterative relations between the time derivative of the $n$-th term in the expansions above and all the lower-order terms. The $\mathcal{O}\left(\alpha^{0}\right)$ terms describe the motion of a free color-charged particle in flat space, i.e. a straight line fixed by the initial momentum, the impact parameter, and initial color charge. The first-order differential equations for the higher-order terms can be integrated; the relevant boundary conditions are that $\boldsymbol{r}_{n \geq 1}, \boldsymbol{p}_{n \geq 1}$ and $c_{i, n \geq 1}^{a}$ vanish at $t=-T$, where $T$ is a large time cutoff. It is necessary to introduce such a cutoff due to the same logarithmic divergence identified in eqs. (2.13) and (2.36). Comparing the result of integrating the color equation of motion to eq. (2.13) it can be shown that the cutoffs $L$ and $T$ are related by $L=2 \beta T$, where $\beta$ is defined in section 2.2. The contribution of each order in $\alpha$ to an observable $O$, such as the linear or color impulse, is then

$$
\Delta O^{(n)}=\int_{-T}^{T} \mathrm{~d} t \frac{\mathrm{d} O^{(n)}}{\mathrm{d} t}=O^{(n)}(t=T)-O^{(n)}(t=-T),
$$

with the complete result being their sum weighted with the appropriate powers of $\alpha$.

\subsection{Hamiltonian from effective field theory}

The perturbative classical problem can be solved straightforwardly once the Hamiltonian is obtained. We then proceed to compute it following the EFT approach adapted to this case. In order to apply this approach we will decompose the amplitudes in some color basis and neglect contributions of higher orders in $\hbar$ using eq. (2.2). Our amplitude expressions will 


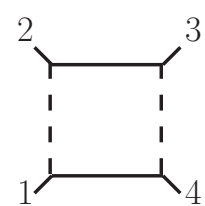

(a)

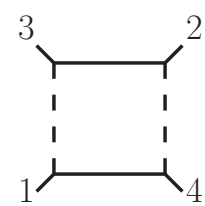

(b)

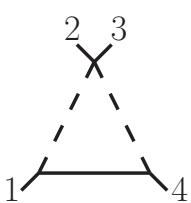

(c)

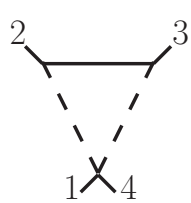

$(d)$

Figure 1. The one-loop scalar box integrals $I_{\square}$ (a) and $I_{\bowtie}$ (b) and the corresponding triangle integrals $I_{\triangle}$ (c) and $I_{\nabla}$ (d). The bottom (top) solid line corresponds to a massive propagator of mass $m_{1}\left(m_{2}\right)$. The dashed lines denote massless propagators.

be directly written in terms of classical color factors, i.e., we consider that the expectation value with respect to coherent states has already been taken ${ }^{8}$.

\subsubsection{Full theory amplitudes from unitarity}

Let us first show the two-to-two scattering amplitudes between color-charged particles needed to construct the Hamiltonian. The information to determine the $\mathcal{O}(\alpha)$ Hamiltonian is contained in the tree-level amplitude. We take the incoming momenta of the colorcharged particles to be $-p_{1}$ and $-p_{2}$ and their outgoing momenta to be $p_{3}$ and $p_{4}$. The amplitude is given by

$$
\mathcal{A}^{\text {tree }}=-\frac{4 \pi \alpha}{q^{2}} \lambda_{1} \mathcal{C}(\succ\langle)+\ldots
$$

where we omit terms that do not contribute to the classical limit in the ellipsis, along with pieces proportional to $q^{2}$, since they cancel the propagator and do not yield long-range contributions. The color structure is given by

$$
\mathcal{C}(\rangle\langle)=c_{1} \cdot c_{2}
$$

and the coefficient $\lambda_{1}$ takes the explicit form

$$
\lambda_{1}=-4 m_{1} m_{2} \sigma,
$$

where we use the kinematic variable

$$
\sigma=\frac{p_{1} \cdot p_{2}}{m_{1} m_{2}}
$$

In order to construct the $\mathcal{O}\left(\alpha^{2}\right)$ Hamiltonian we further need the corresponding oneloop amplitude. It was shown in ref. [3] that classically, the 1-loop scalar YM amplitude has a basis of only one color factor, and moreover depends on the same topologies as in electrodynamics, so it's given by

$$
\mathcal{A}^{1 \text {-loop }}=\mathcal{C}(\neg) \mathcal{A}^{1 \text {-loop, QED }}+\ldots
$$

\footnotetext{
${ }^{8}$ This essentially amounts to the replacement $C_{i} \rightarrow c_{i}$ which is implemented in ref. [3] by the double bracket notation.
} 

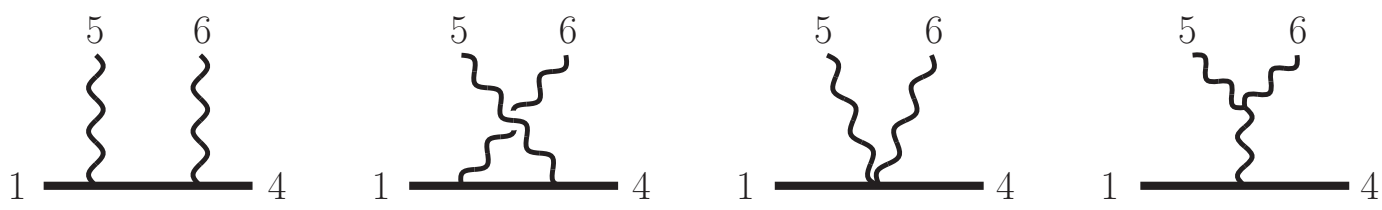

Figure 2. The Compton-amplitude Feynman diagrams. The straight line corresponds to the massive color-charged particle. The wiggly lines correspond to gluons.

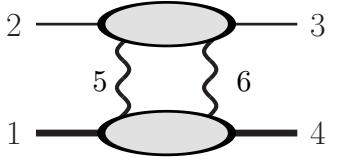

(a)

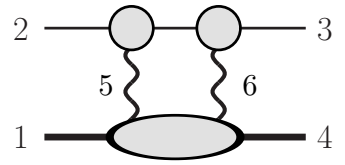

(b)

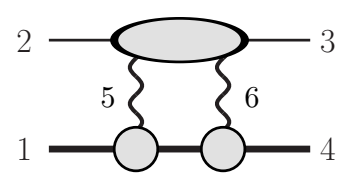

(c)

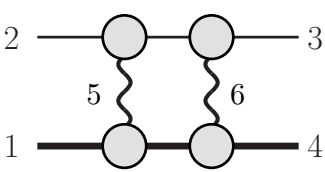

(d)

Figure 3. Appropriate residues of the two-particle cut (a) give the triple cuts (b) and (c), and the quadruple cut (d). The straight lines corresponds to the color-charged particles and the wiggly lines to the exchanged gluons. All exposed lines are taken on-shell.

in terms of the one-loop QED amplitude. The color structure is given by

$$
\mathcal{c}(\mathcal{Z})=\left(c_{1} \cdot c_{2}\right)^{2}
$$

We could express the latter one-loop amplitude as a linear combination of scalar box, triangle, bubble and tadpole integrals, but refs. $[10,13]$ showed that the bubble and tadpole integrals do not contribute to the classical limit. Dropping these pieces we write

$$
i \mathcal{A}^{1-\text { loop, } \mathrm{QED}}=d_{\square} I_{\square}+d_{\bowtie} I_{\bowtie}+c_{\triangle} I_{\triangle}+c_{\nabla} I_{\nabla}
$$

where the coefficients $d_{\square}, d_{\bowtie}, c_{\triangle}$ and $c_{\nabla}$ are rational functions of external momenta. The integrals $I_{\square}, I_{\triangleright}, I_{\triangle}$ and $I_{\nabla}$ are shown in figure 1 . The triangle integrals take the form [10]

$$
I_{\triangle, \nabla}=-\frac{i}{32 m_{1,2}} \frac{1}{\sqrt{-q^{2}}}+\cdots
$$

The box contributions do not contain any novel $\mathcal{O}\left(\alpha^{2}\right)$ information. They correspond to infrared-divergent pieces that cancel out when we equate the full-theory and EFT amplitudes $[10,13]$. In this sense, the explicit values for the box coefficients serve only as a consistency check of our calculation and we do not show them. Instead, we give the result for

$$
i \mathcal{A}^{\triangle+\nabla} \equiv\left(c_{\triangle} I_{\triangle}+c_{\nabla} I_{\nabla}\right) \mathcal{C}(\mathcal{\nearrow}) .
$$

As detailed in ref. [56], we use the generalized-unitarity method to obtain the integral coefficients of eq. (3.14). We start by calculating the Compton amplitude for the colorcharged particle, using Feynman rules. Subsequently, we construct the two-particle cut. The residues of the two-particle cut on the matter poles give the triple cuts, and localizing both matter poles gives the quadruple cut. We obtain the triangle and box coefficients 
from the triple and quadruple cuts respectively. Our result reads

$$
\mathcal{A}^{\triangle+\nabla}=\frac{2 \pi^{2} \alpha^{2}}{\sqrt{-q^{2}}} \lambda_{2} \mathcal{C}(\mathcal{Z})+\ldots
$$

where the coefficient is given by

$$
\lambda_{2}=2 m,
$$

and $m=m_{1}+m_{2}$. In preparation for the matching procedure in the following section, we specialize our expressions to the center-of-mass frame. In this frame, the independent four-momenta read

$$
p_{1}=-\left(E_{1}, \boldsymbol{p}\right), \quad p_{2}=-\left(E_{2},-\boldsymbol{p}\right), \quad q=(0, \boldsymbol{q}), \quad \boldsymbol{p} \cdot \boldsymbol{q}=\boldsymbol{q}^{2} / 2 .
$$

Using the above expressions, our amplitudes take the form

$$
\frac{\mathcal{A}^{\text {tree }}}{4 E_{1} E_{2}}=\frac{4 \pi \alpha}{\boldsymbol{q}^{2}} \Lambda_{1} \mathcal{C}(\rangle\langle), \quad \frac{\mathcal{A}^{\triangle+\nabla}}{4 E_{1} E_{2}}=\frac{2 \pi^{2} \alpha^{2}}{|\boldsymbol{q}|} \Lambda_{2} \mathcal{C}(\not) .
$$

The coefficients $\Lambda_{i}$ are given in terms of the $\lambda_{i}$ of eqs. (3.10) and (3.18) by

$$
\Lambda_{1}=-\frac{\nu \sigma}{\gamma^{2} \xi}, \quad \Lambda_{2}=\frac{1}{2 m \gamma^{2} \xi},
$$

where in addition to the definition in eq. (3.11) we use

$$
\nu=\frac{m_{1} m_{2}}{m^{2}} \quad \gamma=\frac{E}{m}, \quad E=E_{1}+E_{2}, \quad \xi=\frac{E_{1} E_{2}}{E^{2}} .
$$

\subsubsection{Construction of the EFT amplitudes}

With the full theory amplitudes in hand, we now turn our attention to the task of translating the scattering amplitudes of color-charged fields to a two-body conservative Hamiltonian. We do this by matching the scattering amplitude computed above to the two-to-two amplitude of an EFT of the positive-energy modes of fields. Ref. [10] developed this matching procedure for higher orders in the coupling constants and all orders in velocity, and we adapt it here to describe the color-charged fields $\xi_{1}$ and $\xi_{2}$. We follow closely the construction for classical spin in ref. [56]. The action of the effective field theory (supressing representation indices) for $\xi_{1}$ and $\xi_{2}$ is given by

$$
\begin{aligned}
S= & \int \hat{\mathrm{d}}^{D-1} \boldsymbol{k} \sum_{a=1,2} \xi_{a}^{\dagger}(-\boldsymbol{k})\left(i \partial_{t}-\sqrt{\boldsymbol{k}^{2}+m_{a}^{2}}\right) \xi_{a}(\boldsymbol{k}) \\
& -\int \hat{\mathrm{d}}^{D-1} \boldsymbol{k} \int \hat{\mathrm{d}}^{D-1} \boldsymbol{k}^{\prime} \xi_{1}^{\dagger}\left(\boldsymbol{k}^{\prime}\right) \xi_{2}^{\dagger}\left(-\boldsymbol{k}^{\prime}\right) \hat{V}\left(\boldsymbol{k}^{\prime}, \boldsymbol{k}, \hat{\mathcal{C}}_{i}\right) \xi_{1}(\boldsymbol{k}) \xi_{2}(-\boldsymbol{k}),
\end{aligned}
$$

where the interaction potential $\hat{V}\left(\boldsymbol{k}^{\prime}, \boldsymbol{k}, \hat{\mathcal{C}}_{i}\right)$ is a function of the incoming and outgoing momenta $\boldsymbol{k}$ and $\boldsymbol{k}^{\prime}$ and the color-structure operators $\hat{\mathcal{C}}_{i}$. We consider kinematics in the center-of-mass frame. As on the full theory side, one could construct the color asymptotic states of $\xi_{i}$ using $\mathrm{SU}(N)$ coherent states (analogous to the spin coherent states of [56]) 
so color operators satisy the defining properties eqs. (2.5)-(2.6). We obtain the classical color charge vector as the expectation value of the color operator with respect to these on-shell states.

We build the most general potential containing only long-range classical contributions. This will be in terms of color operators, whose expectation values with respect to $\mathrm{SU}(N)$ coherent states are in correspondence with the classical color structures in the full theory amplitude, eq. (3.20). We use the following ansatz for the potential operator

$$
\hat{V}\left(\boldsymbol{k}^{\prime}, \boldsymbol{k}, \hat{\mathcal{C}}_{i}\right)=\frac{4 \pi \alpha}{\hat{\boldsymbol{q}}^{2}} d_{1}\left(\hat{\boldsymbol{p}}^{2}\right) \hat{\mathcal{C}}(\succ \measuredangle)+\frac{2 \pi^{2} \alpha^{2}}{|\hat{\boldsymbol{q}}|} d_{2}\left(\hat{\boldsymbol{p}}^{2}\right) \hat{\mathcal{C}}(\not)+\mathcal{O}\left(\alpha^{3}\right)
$$

where $\hat{\boldsymbol{p}}^{2} \equiv\left(\boldsymbol{k}^{2}+\boldsymbol{k}^{2}\right) / 2$.

We now evaluate the EFT two-to-two scattering amplitude. To this end we use the Feynman rules derived from the EFT action (eq. (3.23)),

$$
\stackrel{(E, \boldsymbol{k})}{\longrightarrow}=\frac{i \rrbracket}{E-\sqrt{\boldsymbol{k}^{2}+m^{2}}+i \epsilon},
$$

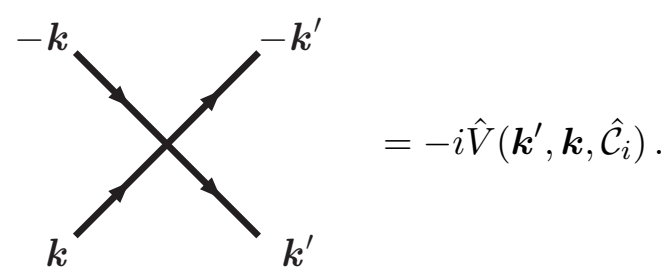

Using these rules we compute the amplitude up to $\mathcal{O}\left(\alpha^{2}\right)$ directly evaluating the relevant Feynman diagrams, omitting terms that do not contribute to long range interactions. The color factors must be treated as operators, and thus their ordering is important. After carrying out the energy integration, we obtain an expression for the amplitude

$$
\hat{\mathcal{A}}^{\mathrm{EFT}}=-\hat{V}\left(\boldsymbol{p}^{\prime}, \boldsymbol{p}, \hat{\mathcal{C}}_{i}\right)-\int \hat{\mathrm{d}}^{D-1} \boldsymbol{k} \frac{\hat{V}\left(\boldsymbol{p}^{\prime}, \boldsymbol{k}, \hat{\mathcal{C}}_{i}\right) \hat{V}\left(\boldsymbol{k}, \boldsymbol{p}, \hat{\mathcal{C}}_{i}\right)}{E_{1}+E_{2}-\sqrt{\boldsymbol{k}^{2}+m_{1}^{2}}-\sqrt{\boldsymbol{k}^{2}+m_{2}^{2}}} .
$$

We now contract the amplitude $\hat{\mathcal{A}}^{\mathrm{EFT}}$ with coherent states, resulting in the operators $\hat{V}$ and $\hat{\mathcal{C}}$ being replaced by their expectation values (hence dropping the hats). At $\mathcal{O}(\alpha)$ the EFT amplitude receives a contribution only from the first term of eq. (3.26). The result is

$$
\mathcal{A}_{\mathcal{O}(\alpha)}^{\mathrm{EFT}}=-\frac{4 \pi \alpha}{\boldsymbol{q}^{2}} d_{1} \mathcal{C}(\succ<)
$$

which is a $c$-number. On the other hand, the EFT amplitude at $\mathcal{O}\left(\alpha^{2}\right)$ receives contributions from both terms in eq. (3.26) and can be written as

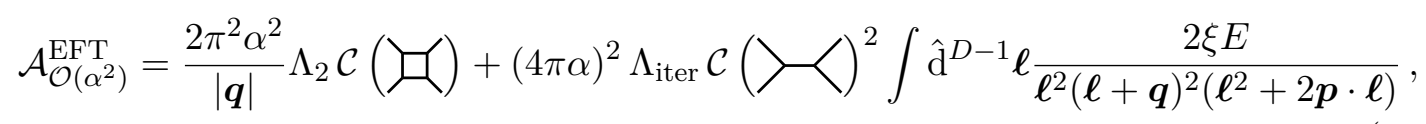

where $\boldsymbol{\ell}=\boldsymbol{k}-\boldsymbol{p}$ and we only keep terms that are relevant in the classical limit. Anticipating the matching, we write the amplitude in terms of $\Lambda_{2}$, which is given directly in terms of the momentum-space potential coefficient by

$$
\Lambda_{2}=-d_{2}+\frac{1-3 \xi}{2 \xi E} d_{1}^{2}+\xi E \partial_{p^{2}} d_{1}^{2}
$$


The second term in eq. (3.28) is infrared divergent and we have explicitly verified that it cancels out when we equate the full-theory and EFT amplitudes. The potential takes the form

$$
V\left(\boldsymbol{r}^{2}, \boldsymbol{p}^{2}, \mathcal{C}_{i}\right)=\frac{\alpha}{|\boldsymbol{r}|} d_{1}\left(\boldsymbol{p}^{2}\right) \mathcal{C}\left(\succ\langle)+\left(\frac{\alpha}{|\boldsymbol{r}|}\right)^{2} d_{2}\left(\boldsymbol{p}^{2}\right) \mathcal{C}(\not)+\mathcal{O}\left(\alpha^{3}\right) .\right.
$$

We obtain the position-space Hamiltonian by taking the Fourier transform of the momentum-space ${ }^{9}$ Hamiltonian with respect to the momentum transfer $\boldsymbol{q}$, which is the conjugate of the separation between the particles $\boldsymbol{r}$. We determine the momentum-space coefficient $d_{i}$ in terms of the amplitudes coefficients $\Lambda_{i}$ by a matching procedure, i.e. by demanding that the EFT amplitude matches the full-theory one,

$$
\mathcal{A}_{\mathcal{O}(\alpha)}^{\mathrm{EFT}}=\frac{\mathcal{A}^{\text {tree }}}{4 E_{1} E_{2}}, \quad \mathcal{A}_{\mathcal{O}\left(\alpha^{2}\right)}^{\mathrm{EFT}}=\frac{\mathcal{A}^{1 \text {-loop }}}{4 E_{1} E_{2}}
$$

where the factors of the energy account for the non-relativistic normalization of the EFT amplitude. Using eq. (3.21) we relate $\Lambda_{i}$ to $\lambda_{i}$, which are explicitly shown in eqs. (3.10) and (3.18). Putting everything together, we obtain expressions for the positionspace coefficients

$$
\begin{aligned}
& d_{1}=-\frac{\nu \sigma}{\gamma^{2} \xi}, \\
& d_{2}=\frac{1}{m \xi}\left(\frac{1}{2 \gamma^{2}}-\frac{\nu \sigma}{\xi \gamma^{3}}+\frac{(1-\xi) \nu^{2} \sigma^{2}}{2 \xi^{2} \gamma^{5}}\right) .
\end{aligned}
$$

This finishes the computation of the effective Hamiltonian. The classical equations of motion can now be solved iteratively using the eqs. (3.3), (3.6) and the definition of the observables (3.7). Following this procedure we have found agreement with the results of section 2 .

\subsection{Observables from the eikonal phase}

The conservative Hamiltonian we obtained in previous sections enables the calculation of physical observables for a scattering of compact objects interacting through gluon exchange. Ref. [56] conjectured a formula that expresses physical observables in terms of derivatives of the eikonal phase for the spinning case. In this section we extend that analysis.

Let us start by obtaining the eikonal phase via a Fourier transform of our amplitudes. Then, following ref. [56] we can solve Hamilton's equations for the impulse and color impulse and relate them to derivatives of the eikonal phase. The eikonal phase $\chi=\chi_{1}+\chi_{2}+\mathcal{O}\left(\alpha^{3}\right)$ is given by

$$
\begin{aligned}
& \chi_{1}=\frac{1}{4 m_{1} m_{2} \sqrt{\sigma^{2}-1}} \int \hat{\mathrm{d}}^{2} \boldsymbol{q} e^{-i \boldsymbol{q} \cdot \boldsymbol{b}} \mathcal{A}^{\mathrm{tree}}(\boldsymbol{q}), \\
& \chi_{2}=\frac{1}{4 m_{1} m_{2} \sqrt{\sigma^{2}-1}} \int \hat{\mathrm{d}}^{2} \boldsymbol{q} e^{-i \boldsymbol{q} \cdot \boldsymbol{b}} \mathcal{A}^{\triangle+\nabla}(\boldsymbol{q}) .
\end{aligned}
$$

\footnotetext{
${ }^{9}$ The position-space coefficients are trivially related to the momentum-space coefficients. This is unlike the case for spinning particles, where a set of linear relations was established between them.
} 
Using our amplitudes expressed in the center-of-mass frame (see eq. (3.20)) we find

$$
\begin{aligned}
& \chi_{1}=-\frac{\xi E \alpha}{|\boldsymbol{p}|} \Lambda_{1}\left(\ln \frac{\boldsymbol{b}^{2}}{L^{2}}\right) \mathcal{C}(\succ\langle), \\
& \chi_{2}=\frac{\pi \xi E \alpha^{2}}{|\boldsymbol{p}|} \frac{\Lambda_{2}}{|\boldsymbol{b}|} \mathcal{C}(\mathcal{C}),
\end{aligned}
$$

where in the first order eikonal phase we include a cutoff regulator $L$ as we did in section 2 . In the case without color, the integration is regulated via dimensional regularization, and the divergence is ignored, because the derivative of the eikonal phase is always taken and they don't contribute. This is no longer the case here.

We may now use the eikonal phase to obtain classical observables. Generalizing the conjecture of ref. [56] to the color-charged case, the observables in question are the impulse $\Delta \boldsymbol{p}$ and color impulse $\Delta c_{i}^{a}$, where

$$
\begin{array}{cl}
\boldsymbol{p}(t=\infty)=\boldsymbol{p}+\Delta \boldsymbol{p}, & \boldsymbol{p}(t=-\infty)=\boldsymbol{p}, \\
c_{i}^{a}(t=\infty)=c_{i}^{a}+\Delta c_{i}^{a}, & c_{i}^{a}(t=-\infty)=c_{i}^{a} .
\end{array}
$$

Inspired by the gravitational spinning case let us decompose the impulse as

$$
\Delta \boldsymbol{p}=\Delta p_{\|} \frac{\boldsymbol{p}}{|\boldsymbol{p}|}+\Delta \boldsymbol{p}_{\perp}
$$

where $\Delta p_{\|}$can be obtained from the on-shell condition $(\boldsymbol{p}+\Delta \boldsymbol{p})^{2}=\boldsymbol{p}^{2}$. Therefore, ignoring the mixing of spin and orbital angular momentum - which is absent in our case since the particle is spinless - the impulse and color impulse through $\mathcal{O}\left(\alpha^{2}\right)$ satisfy

$$
\begin{aligned}
& \Delta \boldsymbol{p}_{\perp}=-\left\{\boldsymbol{p}_{\perp}, \chi\right\}-\frac{1}{2}\left\{\chi,\left\{\boldsymbol{p}_{\perp}, \chi\right\}\right\}, \\
& \Delta c_{1}^{a}=-\left\{c_{1}^{a}, \chi\right\}-\frac{1}{2}\left\{\chi,\left\{c_{1}^{a}, \chi\right\}\right\},
\end{aligned}
$$

where in eq. (3.39) we use the definitions

$$
\left\{\boldsymbol{p}_{\perp}, g\right\} \equiv-\frac{\partial g}{\partial \boldsymbol{b}}, \quad\left\{c_{1}^{a}, g\right\} \equiv f^{a b c} \frac{\partial g}{\partial c_{1}^{b}} c_{1}^{c} .
$$

The second term in the linear impulse doesn't contribute because the tree color structure commutes with itself but we leave it there to keep the suggestive structure. It is then straightforward to show that the linear impulse will be reproduced here, the same way it was for the spinless QED case, simply by taking a replacement of electric for color charges. We have compared both the impulse and the color impulse, to the solution of the equations of motion, and the integrated result of the NLO color impulse finding full agreement.

Our calculation extends the conjecture of ref. [56] to the domain of color. We may note that in this setting the momentum and the color are separately conserved. This is unlike the case for spinning particles, where only the sum $\boldsymbol{J}=\boldsymbol{L}+\boldsymbol{S}$ is conserved. Due to the mixing of spin and orbital angular momentum, it was possible to define the object $\mathcal{D}_{S L}(f, g) \equiv-\boldsymbol{S}_{1} \cdot\left(\frac{\partial f}{\partial \boldsymbol{S}_{1}} \times \frac{\partial g}{\partial \boldsymbol{L}_{b}}\right)$ (where $\boldsymbol{S}_{1}$ is the spin vector and $\boldsymbol{L}_{\boldsymbol{b}} \equiv \boldsymbol{b} \times \boldsymbol{p}$ ). Such an object was necessary to add terms of the form $\mathcal{D}_{S L}(\chi,\{\boldsymbol{o}, \chi\})$ and $\left\{\boldsymbol{o}, \mathcal{D}_{S L}(\chi, \chi)\right\}$. In consequence, the form of eq. (3.39) is indeed simpler than its spin counterpart. 


\section{Conclusions}

In this paper we have used the KMOC formalism and a matching procedure with a nonrelativistic EFT to evaluate classical Yang-Mills observables. Using these approaches we have found that the eikonal phase conjecture of ref. [56] to the case of color is realized at NLO. On the KMOC side we have used the integrands already computed in ref. [3] and performed a direct integration, while on the EFT side we have used unitarity adapting the formalism by Cheung-Rothstein-Solon [10] to the case of color charges.

The integration of the color and momentum impulses follows from a simple integral reduction and techniques successfully applied in gravity, e.g., in ref. [23]. We have found that, as in the case of gravity, the integrals related only with the box and crossed box vanish. However those related with the cut box contribute as expected. In order to expose the exponentiation of the NLO color impulse we have used a cut-off regulator as in ref. [3] to evaluate cut-box integrals.

Once the color decomposition has been performed and the classical relevant parts identified the matching procedure follows essentially the QED case. The Hamiltonian thus constructed was used to solve the equations of motion and obtain the classical linear impulse and color impulse by direct integration. The results were in complete agreement to the evaluation using KMOC integrands. Finally, the eikonal phase construction matches the result of the KMOC and of equations of motion in a rather elegant way giving more evidence of the observation ref. [56] that all physical observables are obtainable via simple manipulations of the scattering amplitude.

For the case of impulses it is also worth mentioning that the intricacies due to the mixing of color and kinematics in the KMOC calculation are absent in the rather straightforward construction based on unitarity and EFT. However, for the construction of the EFT it was crucial to employ coherent states to obtain the classical limit, so this aspect is common to both approaches as is the use of the Lie algebra of the rescaled color factors. Obtaining higher order corrections in the KMOC formalism for Yang-Mills observables would be perhaps more efficient using unitarity from the beginning as done in refs. $[6,7]$ (for the gravitational case), benefiting from advances in relativistic integration.

Our results provide evidence in favor of the eikonal phase conjecture of ref. [56], and so they call for the calculation of the 2-loop color impulse as a toy example towards the gravitational spin. Besides being a toy model for gravitational dynamics, the classical limit of Yang-Mills theory is useful to describe non-equilibrium plasma through kinetic theory, where color is treated as a continuous classical variable. In ref. [8] solutions of kinetic equations were interpreted as classical limits of certain off-shell currents so it would be interesting to explore a Hamiltonian perspective to this problem.

\section{Acknowledgments}

We thank Dimitrios Kosmopoulos for discussions and for sharing code regarding the solution of equations of motion. AL and TS are supported by the U.S. Department of Energy (DOE) under award number DE-SC0009937, and by the Mani L. Bhaumik Institute for 
Theoretical Physics. We thank Donal O'Connell and Radu Roiban for discussions and comments on the manuscript. LDLC acknowledges financial support from the Open Physics Hub at the Physics and Astronomy Department in Bologna. We would like to thank the Galileo Galilei Institute for Theoretical Physics for hosting the workshop on Gravitational scattering, inspiral, and radiation where this collaboration was started. Some of our figures were produced with the help of TikZ-Feynman [98].

Open Access. This article is distributed under the terms of the Creative Commons Attribution License (CC-BY 4.0), which permits any use, distribution and reproduction in any medium, provided the original author(s) and source are credited.

\section{References}

[1] D.A. Kosower, B. Maybee and D. O'Connell, Amplitudes, observables, and classical scattering, JHEP 02 (2019) 137 [arXiv:1811.10950] [INSPIRE].

[2] B. Maybee, D. O'Connell and J. Vines, Observables and amplitudes for spinning particles and black holes, JHEP 12 (2019) 156 [arXiv:1906.09260] [INSPIRE].

[3] L. de la Cruz, B. Maybee, D. O'Connell and A. Ross, Classical Yang-Mills observables from amplitudes, JHEP 12 (2020) 076 [arXiv:2009.03842] [INSPIRE].

[4] A. Cristofoli, R. Gonzo, D.A. Kosower and D. O'Connell, Waveforms from amplitudes, arXiv:2107.10193 [INSPIRE].

[5] A. Manu, D. Ghosh, A. Laddha and P.V. Athira, Soft radiation from scattering amplitudes revisited, JHEP 05 (2021) 056 [arXiv : 2007. 02077] [INSPIRE].

[6] E. Herrmann, J. Parra-Martinez, M.S. Ruf and M. Zeng, Gravitational Bremsstrahlung from reverse unitarity, Phys. Rev. Lett. 126 (2021) 201602 [arXiv:2101.07255] [INSPIRE].

[7] E. Herrmann, J. Parra-Martinez, M.S. Ruf and M. Zeng, Radiative classical gravitational observables at $\mathcal{O}\left(G^{3}\right)$ from scattering amplitudes, JHEP 10 (2021) 148 [arXiv:2104.03957] [INSPIRE].

[8] L. de la Cruz, Scattering amplitudes approach to hard thermal loops, Phys. Rev. D 104 (2021) 014013 [arXiv:2012.07714] [INSPIRE].

[9] D. Neill and I.Z. Rothstein, Classical space-times from the $S$ matrix, Nucl. Phys. B 877 (2013) 177 [arXiv:1304.7263] [INSPIRE].

[10] C. Cheung, I.Z. Rothstein and M.P. Solon, From scattering amplitudes to classical potentials in the post-Minkowskian expansion, Phys. Rev. Lett. 121 (2018) 251101 [arXiv:1808.02489] [INSPIRE].

[11] K. Westpfahl, High-speed scattering of charged and uncharged particles in general relativity, Fortsch. Phys. 33 (1985) 417 [inSPIRE].

[12] Z. Bern, C. Cheung, R. Roiban, C.-H. Shen, M.P. Solon and M. Zeng, Scattering amplitudes and the conservative Hamiltonian for binary systems at third post-Minkowskian order, Phys. Rev. Lett. 122 (2019) 201603 [arXiv:1901.04424] [INSPIRE].

[13] Z. Bern, C. Cheung, R. Roiban, C.-H. Shen, M.P. Solon and M. Zeng, Black hole binary dynamics from the double copy and effective theory, JHEP 10 (2019) 206 [arXiv: 1908.01493] [INSPIRE]. 
[14] C. Cheung and M.P. Solon, Classical gravitational scattering at $\mathcal{O}\left(G^{3}\right)$ from Feynman diagrams, JHEP 06 (2020) 144 [arXiv: 2003.08351] [INSPIRE].

[15] A. Cristofoli, N.E.J. Bjerrum-Bohr, P.H. Damgaard and P. Vanhove, Post-Minkowskian Hamiltonians in general relativity, Phys. Rev. D 100 (2019) 084040 [arXiv:1906.01579] [INSPIRE].

[16] N.E.J. Bjerrum-Bohr, A. Cristofoli and P.H. Damgaard, Post-Minkowskian scattering angle in Einstein gravity, JHEP 08 (2020) 038 [arXiv: 1910.09366] [INSPIRE].

[17] P.H. Damgaard, K. Haddad and A. Helset, Heavy black hole effective theory, JHEP 11 (2019) 070 [arXiv: 1908.10308] [InSPIRE].

[18] R. Aoude, K. Haddad and A. Helset, On-shell heavy particle effective theories, JHEP 05 (2020) 051 [arXiv : 2001.09164] [INSPIRE].

[19] K. Haddad and A. Helset, The double copy for heavy particles, Phys. Rev. Lett. 125 (2020) 181603 [arXiv: 2005.13897] [INSPIRE].

[20] A. Brandhuber, G. Chen, G. Travaglini and C. Wen, A new gauge-invariant double copy for heavy-mass effective theory, JHEP 07 (2021) 047 [arXiv:2104.11206] [INSPIRE].

[21] G. Kälin and R.A. Porto, From boundary data to bound states, JHEP 01 (2020) 072 [arXiv: 1910.03008] [INSPIRE].

[22] G. Kälin and R.A. Porto, From boundary data to bound states. Part II. Scattering angle to dynamical invariants (with twist), JHEP 02 (2020) 120 [arXiv: 1911.09130] [INSPIRE].

[23] G. Kälin and R.A. Porto, Post-Minkowskian effective field theory for conservative binary dynamics, JHEP 11 (2020) 106 [arXiv:2006.01184] [INSPIRE].

[24] G. Kälin, Z. Liu and R.A. Porto, Conservative dynamics of binary systems to third post-Minkowskian order from the effective field theory approach, Phys. Rev. Lett. 125 (2020) 261103 [arXiv:2007.04977] [INSPIRE].

[25] C. Dlapa, G. Kälin, Z. Liu and R.A. Porto, Dynamics of binary systems to fourth post-Minkowskian order from the effective field theory approach, arXiv:2106.08276 [INSPIRE].

[26] G. Mogull, J. Plefka and J. Steinhoff, Classical black hole scattering from a worldline quantum field theory, JHEP 02 (2021) 048 [arXiv: 2010.02865] [INSPIRE].

[27] Z. Bern et al., Scattering amplitudes and conservative binary dynamics at $\mathcal{O}\left(G^{4}\right)$, Phys. Rev. Lett. 126 (2021) 171601 [arXiv:2101.07254] [INSPIRE].

[28] S. Caron-Huot and Z. Zahraee, Integrability of black hole orbits in maximal supergravity, JHEP 07 (2019) 179 [arXiv: 1810.04694] [INSPIRE].

[29] Z. Bern, H. Ita, J. Parra-Martinez and M.S. Ruf, Universality in the classical limit of massless gravitational scattering, Phys. Rev. Lett. 125 (2020) 031601 [arXiv:2002.02459] [INSPIRE].

[30] J. Parra-Martinez, M.S. Ruf and M. Zeng, Extremal black hole scattering at $\mathcal{O}\left(G^{3}\right)$ : graviton dominance, eikonal exponentiation, and differential equations, JHEP 11 (2020) 023 [arXiv: 2005. 04236] [INSPIRE].

[31] M. Carrillo-González, C. de Rham and A.J. Tolley, Scattering amplitudes for binary systems beyond GR, JHEP 11 (2021) 087 [arXiv:2107.11384] [INSPIRE]. 
[32] M.C. Gonzalez, Q. Liang and M. Trodden, Effective field theory for binary cosmic strings, Phys. Rev. D 104 (2021) 043517 [arXiv:2010.15913] [InSPIRE].

[33] F. Loebbert, J. Plefka, C. Shi and T. Wang, Three-body effective potential in general relativity at second post-Minkowskian order and resulting post-Newtonian contributions, Phys. Rev. D 103 (2021) 064010 [arXiv:2012.14224] [INSPIRE].

[34] P. Di Vecchia, C. Heissenberg, R. Russo and G. Veneziano, Universality of ultra-relativistic gravitational scattering, Phys. Lett. B 811 (2020) 135924 [arXiv:2008.12743] [INSPIRE].

[35] P. Di Vecchia, C. Heissenberg, R. Russo and G. Veneziano, Radiation reaction from soft theorems, Phys. Lett. B 818 (2021) 136379 [arXiv:2101.05772] [InSPIRE].

[36] T. Damour, Radiative contribution to classical gravitational scattering at the third order in G, Phys. Rev. D 102 (2020) 124008 [arXiv:2010.01641] [InSPIRE].

[37] G.U. Jakobsen, G. Mogull, J. Plefka and J. Steinhoff, Classical gravitational Bremsstrahlung from a worldline quantum field theory, Phys. Rev. Lett. 126 (2021) 201103 [arXiv:2101.12688] [INSPIRE].

[38] S. Mougiakakos, M.M. Riva and F. Vernizzi, Gravitational Bremsstrahlung in the post-Minkowskian effective field theory, Phys. Rev. D 104 (2021) 024041 [arXiv: 2102.08339] [INSPIRE].

[39] N.E.J. Bjerrum-Bohr, P.H. Damgaard, L. Planté and P. Vanhove, The amplitude for classical gravitational scattering at third post-Minkowskian order, arXiv:2105.05218 [INSPIRE].

[40] K. Haddad and A. Helset, Tidal effects in quantum field theory, JHEP 12 (2020) 024 [arXiv:2008.04920] [INSPIRE].

[41] R. Aoude, K. Haddad and A. Helset, Tidal effects for spinning particles, JHEP 03 (2021) 097 [arXiv: 2012.05256] [INSPIRE].

[42] M. Accettulli Huber, A. Brandhuber, S. De Angelis and G. Travaglini, From amplitudes to gravitational radiation with cubic interactions and tidal effects, Phys. Rev. D 103 (2021) 045015 [arXiv: 2012.06548] [INSPIRE].

[43] G. Kälin, Z. Liu and R.A. Porto, Conservative tidal effects in compact binary systems to next-to-leading post-Minkowskian order, Phys. Rev. D 102 (2020) 124025 [arXiv: 2008.06047] [INSPIRE].

[44] C. Cheung and M.P. Solon, Tidal effects in the post-Minkowskian expansion, Phys. Rev. Lett. 125 (2020) 191601 [arXiv:2006.06665] [INSPIRE].

[45] C. Cheung, N. Shah and M.P. Solon, Mining the geodesic equation for scattering data, Phys. Rev. D 103 (2021) 024030 [arXiv: 2010.08568] [InSPIRE].

[46] Z. Bern, J. Parra-Martinez, R. Roiban, E. Sawyer and C.-H. Shen, Leading nonlinear tidal effects and scattering amplitudes, JHEP 05 (2021) 188 [arXiv:2010.08559] [INSPIRE].

[47] B.R. Holstein and A. Ross, Spin effects in long range gravitational scattering, arXiv:0802.0716 [INSPIRE].

[48] V. Vaidya, Gravitational spin Hamiltonians from the S matrix, Phys. Rev. D 91 (2015) 024017 [arXiv: 1410.5348] [INSPIRE].

[49] A. Guevara, Holomorphic classical limit for spin effects in gravitational and electromagnetic scattering, JHEP 04 (2019) 033 [arXiv:1706.02314] [INSPIRE]. 
[50] A. Guevara, A. Ochirov and J. Vines, Scattering of spinning black holes from exponentiated soft factors, JHEP 09 (2019) 056 [arXiv: 1812.06895] [INSPIRE].

[51] M.-Z. Chung, Y.-T. Huang, J.-W. Kim and S. Lee, The simplest massive S-matrix: from minimal coupling to black holes, JHEP 04 (2019) 156 [arXiv:1812.08752] [INSPIRE].

[52] M.-Z. Chung, Y.-T. Huang and J.-W. Kim, Classical potential for general spinning bodies, JHEP 09 (2020) 074 [arXiv: 1908.08463] [INSPIRE].

[53] M.-Z. Chung, Y.-t. Huang, J.-W. Kim and S. Lee, Complete Hamiltonian for spinning binary systems at first post-Minkowskian order, JHEP 05 (2020) 105 [arXiv: 2003.06600] [INSPIRE].

[54] A. Guevara, A. Ochirov and J. Vines, Black-hole scattering with general spin directions from minimal-coupling amplitudes, Phys. Rev. D 100 (2019) 104024 [arXiv:1906.10071] [INSPIRE].

[55] J. Vines, Scattering of two spinning black holes in post-Minkowskian gravity, to all orders in spin, and effective-one-body mappings, Class. Quant. Grav. 35 (2018) 084002 [arXiv: 1709.06016] [INSPIRE].

[56] Z. Bern, A. Luna, R. Roiban, C.-H. Shen and M. Zeng, Spinning black hole binary dynamics, scattering amplitudes, and effective field theory, Phys. Rev. D 104 (2021) 065014 [arXiv: 2005. 03071] [INSPIRE].

[57] Z. Liu, R.A. Porto and Z. Yang, Spin effects in the effective field theory approach to post-Minkowskian conservative dynamics, JHEP 06 (2021) 012 [arXiv:2102.10059] [INSPIRE].

[58] D. Kosmopoulos and A. Luna, Quadratic-in-spin Hamiltonian at $\mathcal{O}\left(G^{2}\right)$ from scattering amplitudes, JHEP 07 (2021) 037 [arXiv:2102.10137] [INSPIRE].

[59] G.U. Jakobsen, G. Mogull, J. Plefka and J. Steinhoff, Gravitational bremsstrahlung and hidden supersymmetry of spinning bodies, arXiv:2106.10256 [INSPIRE].

[60] B.-T. Chen, M.-Z. Chung, Y.-t. Huang and M.K. Tam, Minimal spin deflection of Kerr-Newman and supersymmetric black hole, arXiv:2106.12518 [INSPIRE].

[61] Y.F. Bautista, A. Guevara, C. Kavanagh and J. Vines, From scattering in black hole backgrounds to higher-spin amplitudes: part I, arXiv:2107.10179 [INSPIRE].

[62] R. Aoude and A. Ochirov, Classical observables from coherent-spin amplitudes, JHEP 10 (2021) 008 [arXiv: 2108.01649] [INSPIRE].

[63] M. Chiodaroli, H. Johansson and P. Pichini, Compton black-hole scattering for $s \leq 5 / 2$, arXiv:2107.14779 [INSPIRE].

[64] D. Amati, M. Ciafaloni and G. Veneziano, Higher order gravitational deflection and soft Bremsstrahlung in Planckian energy superstring collisions, Nucl. Phys. B 347 (1990) 550 [INSPIRE].

[65] S. Melville, S.G. Naculich, H.J. Schnitzer and C.D. White, Wilson line approach to gravity in the high energy limit, Phys. Rev. D 89 (2014) 025009 [arXiv: 1306.6019] [inSPIRE].

[66] A. Luna, S. Melville, S.G. Naculich and C.D. White, Next-to-soft corrections to high energy scattering in QCD and gravity, JHEP 01 (2017) 052 [arXiv:1611.02172] [INSPIRE].

[67] R. Akhoury, R. Saotome and G. Sterman, High energy scattering in perturbative quantum gravity at next to leading power, Phys. Rev. D 103 (2021) 064036 [arXiv:1308.5204] [INSPIRE]. 
[68] A. Koemans Collado, P. Di Vecchia and R. Russo, Revisiting the second post-Minkowskian eikonal and the dynamics of binary black holes, Phys. Rev. D 100 (2019) 066028 [arXiv: 1904.02667] [INSPIRE].

[69] A. Cristofoli, P.H. Damgaard, P. Di Vecchia and C. Heissenberg, Second-order post-Minkowskian scattering in arbitrary dimensions, JHEP 07 (2020) 122 [arXiv: 2003.10274] [INSPIRE].

[70] P. Di Vecchia, A. Luna, S.G. Naculich, R. Russo, G. Veneziano and C.D. White, A tale of two exponentiations in $\mathcal{N}=8$ supergravity, Phys. Lett. B 798 (2019) 134927 [arXiv: 1908.05603] [INSPIRE].

[71] P. Di Vecchia, S.G. Naculich, R. Russo, G. Veneziano and C.D. White, A tale of two exponentiations in $\mathcal{N}=8$ supergravity at subleading level, JHEP 03 (2020) 173 [arXiv: 1911.11716] [INSPIRE].

[72] P. Di Vecchia, C. Heissenberg, R. Russo and G. Veneziano, The eikonal approach to gravitational scattering and radiation at $\mathcal{O}\left(G^{3}\right)$, JHEP 07 (2021) 169 [arXiv:2104.03256] [INSPIRE].

[73] C. Heissenberg, Infrared divergences and the eikonal exponentiation, Phys. Rev. D 104 (2021) 046016 [arXiv:2105.04594] [INSPIRE].

[74] P.H. Damgaard, L. Plante and P. Vanhove, On an exponential representation of the gravitational S-matrix, JHEP 11 (2021) 213 [arXiv:2107.12891] [INSPIRE].

[75] J. Vines, J. Steinhoff and A. Buonanno, Spinning-black-hole scattering and the test-black-hole limit at second post-Minkowskian order, Phys. Rev. D 99 (2019) 064054 [arXiv:1812.00956] [INSPIRE].

[76] N. Siemonsen and J. Vines, Test black holes, scattering amplitudes and perturbations of Kerr spacetime, Phys. Rev. D 101 (2020) 064066 [arXiv: 1909.07361] [InSPIRE].

[77] Z. Bern, J.J. Carrasco, M. Chiodaroli, H. Johansson and R. Roiban, The duality between color and kinematics and its applications, arXiv:1909.01358 [INSPIRE].

[78] W.D. Goldberger and A.K. Ridgway, Radiation and the classical double copy for color charges, Phys. Rev. D 95 (2017) 125010 [arXiv:1611.03493] [INSPIRE].

[79] W.D. Goldberger, S.G. Prabhu and J.O. Thompson, Classical gluon and graviton radiation from the bi-adjoint scalar double copy, Phys. Rev. D 96 (2017) 065009 [arXiv:1705.09263] [INSPIRE].

[80] W.D. Goldberger and A.K. Ridgway, Bound states and the classical double copy, Phys. Rev. D 97 (2018) 085019 [arXiv: 1711.09493] [INSPIRE].

[81] W.D. Goldberger, J. Li and S.G. Prabhu, Spinning particles, axion radiation, and the classical double copy, Phys. Rev. D 97 (2018) 105018 [arXiv:1712.09250] [INSPIRE].

[82] D. Chester, Radiative double copy for Einstein-Yang-Mills theory, Phys. Rev. D 97 (2018) 084025 [arXiv: 1712.08684 ] [INSPIRE].

[83] C.-H. Shen, Gravitational radiation from color-kinematics duality, JHEP 11 (2018) 162 [arXiv: 1806.07388] [INSPIRE].

[84] A. Luna et al., Perturbative spacetimes from Yang-Mills theory, JHEP 04 (2017) 069 [arXiv: 1611.07508] [INSPIRE]. 
[85] A. Luna, I. Nicholson, D. O'Connell and C.D. White, Inelastic black hole scattering from charged scalar amplitudes, JHEP 03 (2018) 044 [arXiv:1711.03901] [INSPIRE].

[86] M. Carrillo González, R. Penco and M. Trodden, Radiation of scalar modes and the classical double copy, JHEP 11 (2018) 065 [arXiv:1809.04611] [INSPIRE].

[87] J. Plefka, J. Steinhoff and W. Wormsbecher, Effective action of dilaton gravity as the classical double copy of Yang-Mills theory, Phys. Rev. D 99 (2019) 024021 [arXiv: 1807.09859] [INSPIRE].

[88] J. Plefka, C. Shi, J. Steinhoff and T. Wang, Breakdown of the classical double copy for the effective action of dilaton-gravity at NNLO, Phys. Rev. D 100 (2019) 086006 [arXiv: 1906.05875] [INSPIRE].

[89] R. Monteiro, D. O'Connell and C.D. White, Black holes and the double copy, JHEP 12 (2014) 056 [arXiv: 1410.0239] [INSPIRE].

[90] A. Luna, R. Monteiro, I. Nicholson and D. O'Connell, Type D spacetimes and the Weyl double copy, Class. Quant. Grav. 36 (2019) 065003 [arXiv:1810.08183] [INSPIRE].

[91] C. Keeler, T. Manton and N. Monga, From Navier-Stokes to Maxwell via Einstein, JHEP 08 (2020) 147 [arXiv: 2005. 04242] [INSPIRE].

[92] C. Cheung and J. Mangan, Scattering amplitudes and the Navier-Stokes equation, arXiv:2010.15970 [INSPIRE].

[93] N.E.J. Bjerrum-Bohr, P.H. Damgaard, G. Festuccia, L. Planté and P. Vanhove, General relativity from scattering amplitudes, Phys. Rev. Lett. 121 (2018) 171601 [arXiv: 1806.04920] [INSPIRE].

[94] N.E.J. Bjerrum-Bohr, P.H. Damgaard, L. Planté and P. Vanhove, Classical gravity from loop amplitudes, Phys. Rev. D 104 (2021) 026009 [arXiv: 2104.04510] [INSPIRE].

[95] S.K. Wong, Field and particle equations for the classical Yang-Mills field and particles with isotopic spin, Nuovo Cim. A 65 (1970) 689 [INSPIRE].

[96] A.P. Balachandran, P. Salomonson, B.-S. Skagerstam and J.-O. Winnberg, Classical description of particle interacting with non-Abelian gauge field, Phys. Rev. D 15 (1977) 2308 [INSPIRE].

[97] A.P. Balachandran, S. Borchardt and A. Stern, Lagrangian and Hamiltonian descriptions of Yang-Mills particles, Phys. Rev. D 17 (1978) 3247 [INSPIRE].

[98] J. Ellis, TikZ-Feynman: Feynman diagrams with TikZ, Comput. Phys. Commun. 210 (2017) 103 [arXiv: 1601.05437 ] [INSPIRE]. 